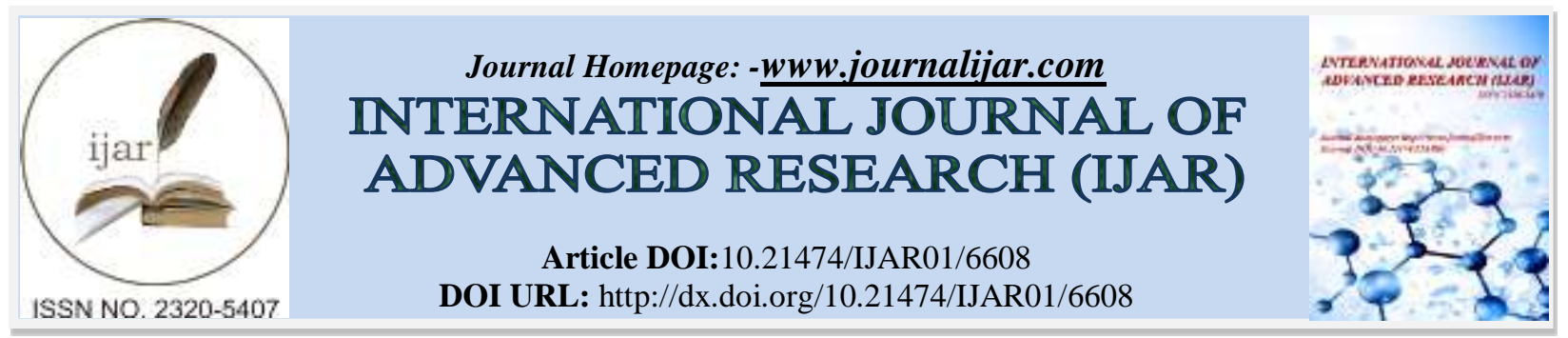

RESEARCH ARTICLE

\title{
THE ALTERATIONS OF PLATELET COUNTS AND PT-INR LEVELS AFTER RIGHT HEPATECTOMY AND SAFE ANTICOAGULANT PROPHYLAXIS.
}

\author{
Sinan Hatipoglu ${ }^{1,2}$ and Cuneyt Kayaalp ${ }^{2}$. \\ 1. Department of Surgery Unit, School of Medicine, Adiyaman University, Adiyaman, Turkey. \\ 2. Liver Transplant Institute, School of Medicine, Inonu University, Malatya, Turkey.
}

\section{Manuscript Info}

Manuscript History

Received: 22 December 2017

Final Accepted: 24 January 2018

Published: February 2018

Keywords:-

Anticoagulants, Blood Coagulation

Tests, Surgical Hemostasis, Right

Hepatectomy, Liver Transplantation.

\section{Abstract}

Background: Anticoagulant prophylaxis for major hepatectomy operations is still unclear. Liver plays a main role on hemostasis and coagulation parameters are disturbed by major hepatectomies. The risk of thromboembolism increases after the hepatectomies as in other major abdominal operations. However, presence of anticoagulant prophylaxis constitute a significant risk of bleeding complications. Here, we aimed to interpret the effects of donor hepatectomies on the coagulation functions and to evaluate the role of safe anticoagulant prophylaxis.

Material and Methods: The retrospective data included the healthy 100 adult patients who underwent right-lobe donor hepatectomy (RLDH) for living donor liver transplantation (LDLT). Anticoagulant prophylaxis was applied none of these patients. Perioperative hematological laboratory tests for 3 days and the incidence of postoperative clinical thromboembolic events were evaluated.

Results: The mean age was 30.8 (ranged 18-61) and 45 of them were women. Hemoglobin, hematocrit and platelet values decreased till postoperative day $2(\mathrm{p}<0.05)$ and started to increase on day 3. PT, aPTT and INR values increased till postoperative day 2 and trend to be normal on day 3. There was no thrombo-embolic event. All of the donors remained alive with normal liver functions.

Conclusions: Major liver resections, particularly RLDH, are different from other operations of general surgery with their effect on coagulation cascade. Coagulation abnormalities that may be seen in proportion with the liver resection must be taken into consideration in donors and routine anticoagulant treatment protocols might be reviewed when the postoperative bleeding is most hazardous. Patients who have the risk of postoperative thromboembolic complications should be reassessed after the postoperative second day.

Copy Right, IJAR, 2018,. All rights reserved.

\section{Introduction:-}

Nowadays, major liver resections (MLR) are widely performed for the treatment of various malignant or benign liver tumors and living donor liver transplantations (LDLT). Major liver resections have developed dramatically 
with improved understanding of the anatomic segments of the liver, enhanced imaging by triphasic liver computerized tomography and magnetic resonance imaging scans, improved of anesthesia and transplant surgery, enhanced critical care, postoperative nursing and physical therapy, and technological advances in surgical devices $(1,2)$.

Right lobe liver resection (RLLR) is especially challenging because of its original and complex anatomic structure with major volume and because of its vital functions such as hemostasis functions. The liver plays a significant role in maintaining hemostasis function and it produces almost all the factors for coagulation and fibrinolysis. The liver reticuloendothelial system also plays a major role in disposing activated coagulation and fibrinolysis-related factors and inhibitors (3).

After the MLR such as RLLR, the production of coagulation and fibrinolysis-related factors is diminished, as well as its inhibitors. Furthermore, the hematological profile is complicated by a decreased rate of clearance of activated clotting factors, thrombocytopenia induced by liver resection, intra-operative bleeding or fluid administration, and some quantitative and qualitative abnormalities of the coagulation factors. This is an important topic, namely the risk of hemorrhage in elective liver resection vs the risk of a hypercoagulable state and a thrombotic complication. In this article, we aimed to interpret effects of RLLR on the coagulation functions and evaluate safe anticoagulant prophylaxis approach in the live donor patient (LDP) group.

\section{Material and Methods:-}

Data from medical records of healthy adult patients that underwent RLLR for LDLT were reviewed for the study between February and October 2011. All of the LDP were identified by retrospective review that contained donor demographics data, BMI (body mass index), weight of liver grafts, GWDW (Graft weight/donor weight ratio) and the laboratory test values of preoperative and postoperative data on coagulation profile on postoperative days (POD)Oto POD 3 were collected ( hemoglobin (Hgb), hematocrit (Htc), platelet counts (Plt), prothrombin time (PT), active partial thromboplastin time (aPTT) and international normalized ratio (INR) ). The volume of blood loss and intra-operative fluids administered was recorded. Intra-operative blood loss was calculated and the blood estimated to be contained in cotton swabs and gauzes minus the recorded volume of fluid used to flush the surgical space and in suction system.

Live donors who required peri-operative blood or blood product transfusions, re-operative procedures and patients who received anti-platelet drugs, anticoagulant therapy or prophylaxis were all excluded. We did not use heparin in the live donors just before taking the graft out. Prothrombin time, INR, aPTT, and platelet count level should be tested to aid in making the diagnosis of a postoperative coagulation disorder. A coagulation disorder was defined as one or more of the following events postoperatively: peak PT $>1.5$ INR (INR; highest quartile of PT), peak aPTT $>46$ seconds (highest quartile), or nadir platelet count $<100 \times 10^{9} / \mathrm{L}$ (4).

\section{Donor selection:-}

Adequate selection of LDP is a major prerequisite for right lobe-LDLT. Donor selection process for right lobeLDLT is a comprehensive and labor-intensive multidisciplinary process. Both include medical, psychosocial and surgical-anatomic evaluations. Each donor was related to the recipient, was selected from a strictly voluntary pool. The investigation protocol for donors included serum electrolyte levels, standard liver function tests, enzyms, full blood analysis, coagulation tests, serology tests for HIV and hepatitis B and C viruses, electrocardiography, chest radiography, multi-slice spiral computed tomography were performed. Serology for viral hepatitis was negative, and ABO blood group compatibility was mandatory. Volumetric assessment of the right lobe liver graft, total liver volumes, liver steatosis ratio and vascular anatomy are evaluated with computerized tomographic angiography by radiology department. Donors with graft-to-recipient weight ratios $>0.8 \%$ and liver steatosis $<30 \%$ were accepted for transplantation. Multiple arteries, bile ducts, and various types of portal veins in the donor liver were not considered to be contraindications for donation. Finally, informed consent was obtained from the volunteer donor in the absence of other family members. All donor cases were evaluated and approved by independent institutional committees.

\section{Surgical Prosedure:-}

The donor surgery was started firstly for LDLT. After a J-shaped skin incision, the donor liver had been inspected and judged to be satisfactory, the recipient surgery was started to minimize the cold ischemia time. The RLLR procedure was planned according to GWDW, hepatic vein, portal vein, and hepatic artery anatomy. The RLLR was 
carried out using a Cavitron Ultrasonic Surgical Aspirator system (CUSA Excel' ${ }^{\mathrm{TM}}$, Valleylab Inc., Boulder, Colo) and electrocautery. Cholecystectomy and intra-operative cholangiogram were performed to delineate any biliary system anomaly, especially the variation in the drainage of the right posterior segment duct. The Pringle maneuver was not used routinely in the donors during parenchymal transaction and vascular inflow and outflow were not interrupted during RLLR. All of RLLR underwent a standard anesthetic technique with monitoring devices, of continuous electrocardiography, pulse oximetry, capnography, arterial line for blood pressure monitoring, and nasopharyngeal temperature. After the RLLR, all LDP were transferred to the surgical intensive care unit for appropriate post-operative observation and treatment.

\section{Statistical Analyses:-}

Continuous variables are reported as means \pm standard deviations, and categorical variables as numbers and percentages. Data were compared with chi-square test or Fisher's exact test 2 tailed for categorical variables and Student's t-test for continuous variables; $\mathrm{p}<0.05$ were considered significant. Data were analyzed using the SPSS (Statistical Package for Social Sciences) 9.05 for Windows ${ }^{\circledR}$ software.

\section{Results:-}

We had performed a hundred RLLR during the stated study period in our institution. All of the donors were classified as ASA physical status I-II and had no history of liver disease. Donor demographic characteristics as well as surgical and coagulation values data are shown in Tables 1.The median age was $30.8 \pm 8.9$ (range 18-61) years and 45 of the patients were women, whereas 55 were men. The mean weight of the patients was $67.5 \pm 10.1$ kilogram and the mean weight of right lobe graft was $718.9 \pm 169.7$ gram. Mean operation time was $261 \pm 76$ minutes. Intra-operative blood loss and intra-operative infused fluids were observed similar to each patients in our group ( $\mathrm{p}>0.05)$.

Table 1:- Demographic and Intraoperative Data of the a Hundred Live Donors

\begin{tabular}{|lc|}
\hline Age (years) & $30.8 \pm 8.9(18-61)$ \\
\hline Gender $(\mathbf{m a l e} / \mathbf{f e m a l e})$ & $55 / 45$ \\
\hline Length $(\mathbf{c m})$ & $167.25 \pm 8.14$ \\
\hline Body weight $\mathbf{( k g )}$ & $67.5 \pm 10.1$ \\
\hline Body Mass Index $\left(\mathbf{k g} / \mathbf{m}^{2}\right)$ & $23.63(18.67-34.03)$ \\
\hline Weight of Right-Lobe Liver Graft $(\mathbf{g r})$ & $718.9 \pm 169.7$ \\
\hline Operation time $(\mathbf{m i n})$ & $261 \pm 76$ \\
\hline Total infused fluid $(\mathbf{m L})$ & $5462 \pm 921$ \\
\hline Estimated blood loss $(\mathbf{m L})$ & $535 \pm 213$ \\
\hline Postoperative hospital stay (day) & $5.5 \pm 1.7$ \\
\hline
\end{tabular}

Note: Values are presented as median (range), mean \pm standard deviation or numbers of donors.

Hemoglobin, hematocrit and thrombocyte counts showed statistically significant $(\mathrm{p}<0.05)$ declines until the postoperative day 2, however the values started to increase at the day 3 (Figure 1A, 1B, 1C). Prothrombin time, aPTT and INR values showed statistically significant $(\mathrm{p}<0.05)$ increments until the postoperative day 2 , but at the day 3 the values had returned to normal (Figure 1D, 1E, 1F).The proportion of patients having an abnormal coagulation profile peaked on POD 2 (Figure 1). The patients had experienced no major complications as thrombosis-embolism and/or bleeding. No prophylactic blood product replacement and no anticoagulant prophylaxis-treatment were applied to the patients. All of the donors remained alive with normal liver functions at the time of the final follow-up examination. 

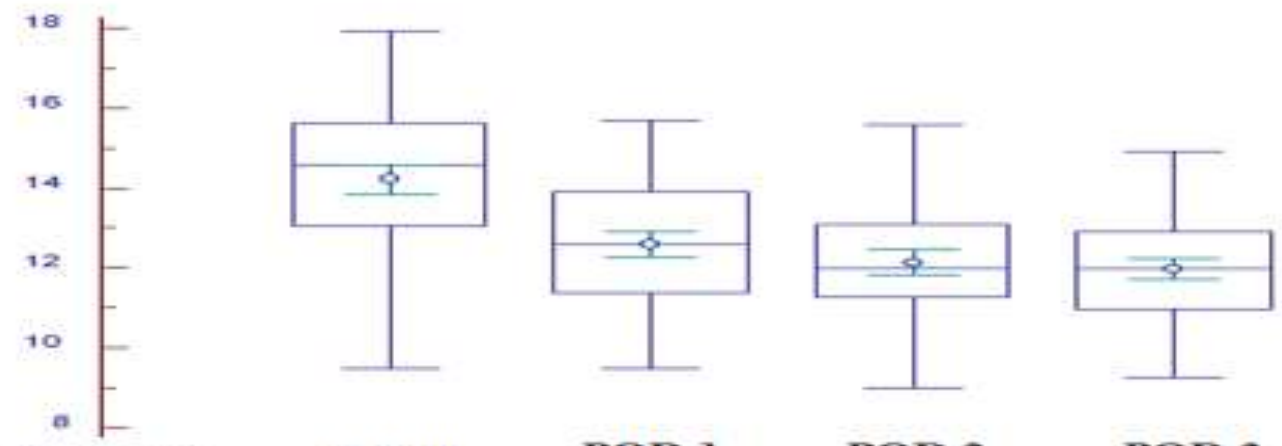

Heb ca/al pre-op

POD 1

POD 2

POD 3
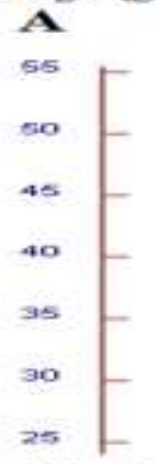

Hre(e)

I3
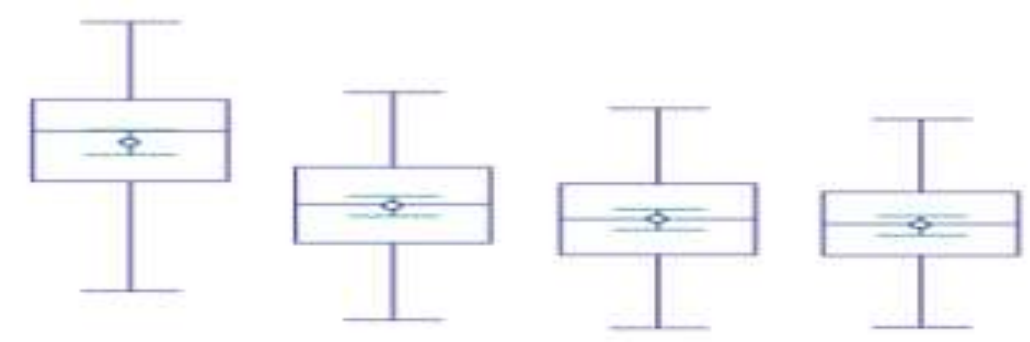

pro op

POD 1

POD 2

POD 3

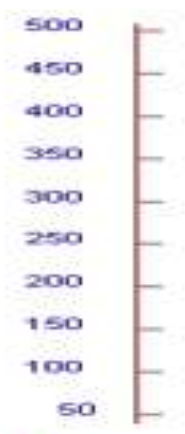

Ple $\left(10^{3 / \mathrm{ml}}\right)$

$c$
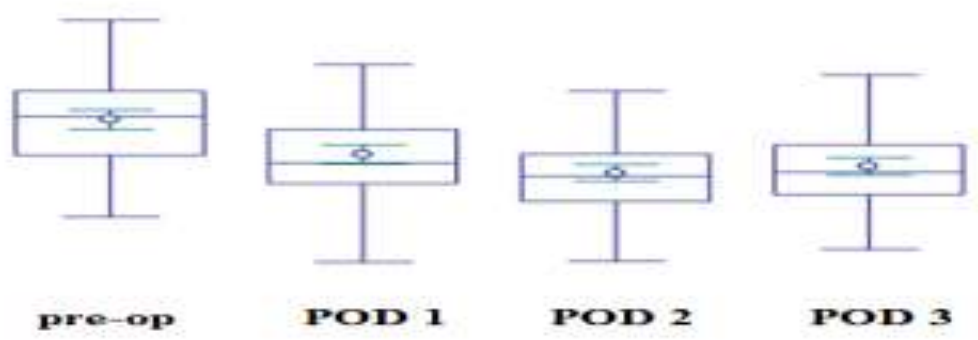

POD 1

POD 2

POD 3

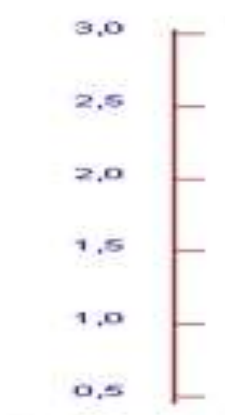

INTR

D
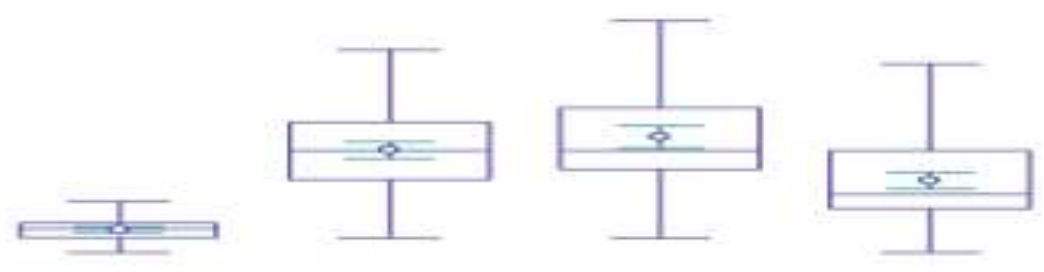

pre-op

POD 1

POS 2

POr 3 


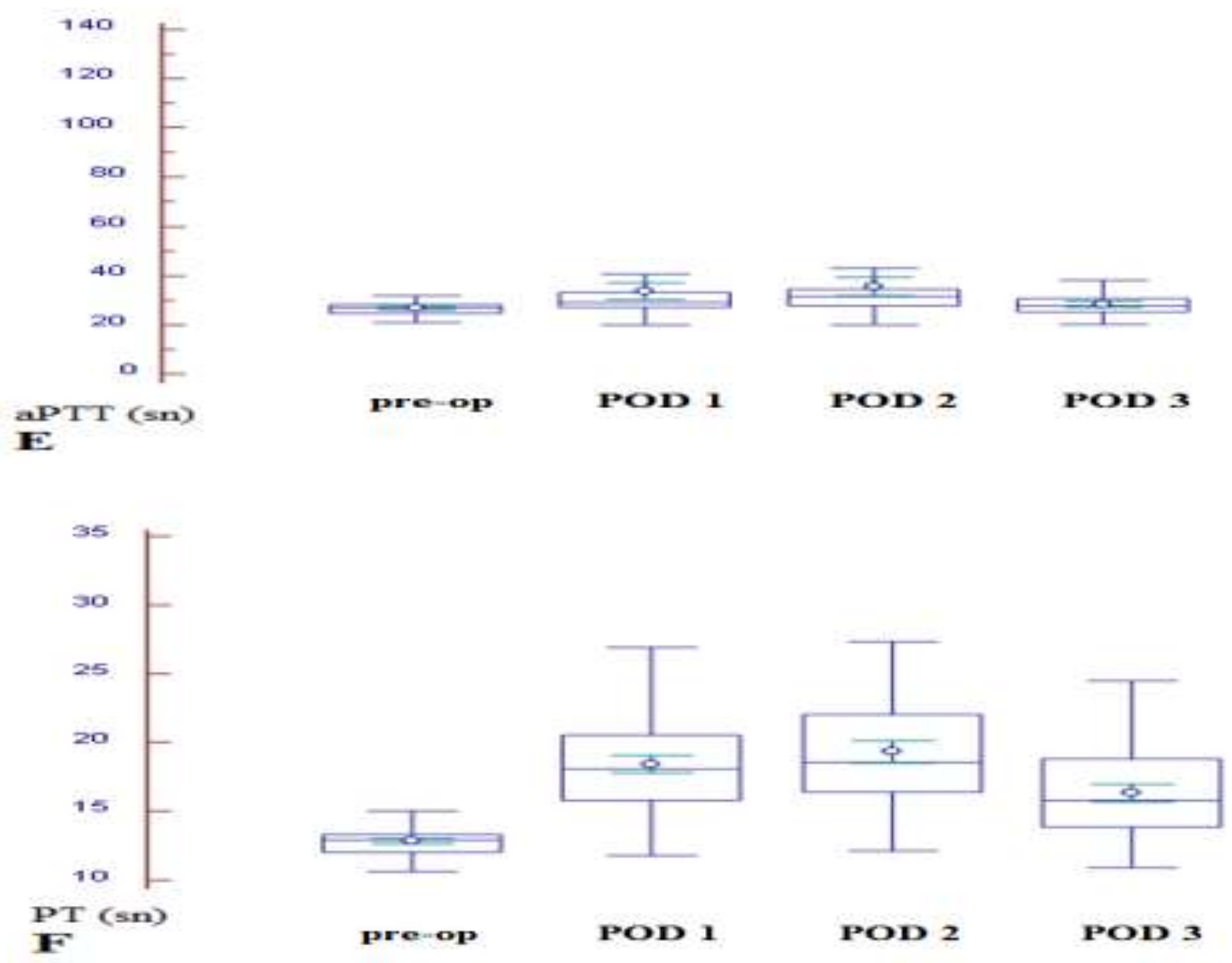

Figure 1:- Changes in peri-operative biochemical and hematologic laboratory values for the hemoglobin (Hgb)-(Figure 1A), hematocrit (Htc)-(Figure 1B), platelet counts (PIt)-(Figure 1C), international normalized ratio (INR)-(Figure 1D), activated partial thromboplastin time (aPTT)-(Figure 1E), and prothrombin time (PT)-(Figure 1F) in 100 livedonors. The middle lines represent the medians. POD: Post operative day, pre-op: Pre operative period.

\section{Discussion:-}

Living donor liver transplantation had an impressive growth over time especially in our country (5-7). The shortage of cadaveric organs and the high number of patients waiting for a liver transplantation have caused an increasing interest in LDLT. Right lobe-LDLT is an excellent option for adult patients with end stage liver failure. Minimizing the mortality and morbidity rates with increasing number of LDLT depends on the absence of serious deteriorations in the remnant liver functions. The surgical risks of RLLR need to be carefully evaluated for LDP and considered with care to maximize donor safety.

Despite of technical advances in liver transplant surgery and high experience of liver resection of specialized centers and, it is still burdened by relatively high rates of post-operative morbidity $(4.09 \%-47.7 \%)$ and mortality $(0.24 \%$ $9.7 \%)$, (8). Coagulopathy is one of the main problems in patients undergoing RLLR. Living donor patients with a normal preoperative coagulation profile may suffer from transient postoperative coagulation disorder by RLLR. The complications of RLLR due to hemostasis disorders comprise intraperitoneal hemorrhage, gastrointestinal tract bleeding, biliary tract hemorrhage, coagulation disorders, thrombosis-embolism, acute liver failure, and small for size syndrome (8).

The common causes of coagulation disorders associated with RLLR are respectively; volumetric failure of the remnant liver due to massive RLLR, functional failure of the remnant liver due to vascular pahthologies and prolonged ischemia, the coagulopathy is related to a decrease in clotting factors and platelets caused by surgical massive intra-operative hemorrhage and/or blood transfusion of more than $4000 \mathrm{~mL}$, administration of large volumes of crystalloid, colloid, or blood products, and overdose of heparin after hepatic artery or portal vein 
catheterization $(1,8-12)$. The liver plays a major role in the clotting process, and after the RLLR, remnant liver may consist as characterized by decreased synthesis of among all clotting and inhibitor factors, decreased clearance of activated factors, decreased plasminogen activator inhibitor factors, and simultaneous generation of tissue plasminogen activator, quantitative and qualitative platelet defects, hyperfibrinolysis, and accelerated intravascular coagulation (11-13). Remnant liver volume is nearly $30 \%$ to $40 \%$ of the liver volume, which often causes transient alterations in liver functions as well as coagulation profiles at the end of the operation and during the postoperative period $(14,15)$. So that, small remnant liver volume may increase the high risk of coagulation imbalance and the bleeding tendency accounts for increased risk of morbidity and mortality in patients with RLLR (13).

Our study shows that remarkable alterations in Hgb, Htc, Plt, INR, PT and aPTT values were observed in a hundred LDP who underwent uncomplicated RLLR without anticoagulant prophylaxis and treatment. We observed that increased in PT, INR and aPTT and decreased in Hgb, Htc, and platelet count developed after RLLR (Figure 1). Postoperative coagulation imbalance has been well investigated after donor hepatectomy (16-20). A elongated PT is a common finding after MLR and PT required 3-6 days to return to normal levels because of transiently impaired hepatic synthesis $(16,17)$. Prothrombin time is related both to increased bleeding risk and mortality; patients with a PT prolongation of more than 1.5 seconds and more than 2.5 seconds have mortality rates of $47 \%$ and $87 \%$, respectively versus 7\% for patients with normal PT (13). A PT prolonged for more than 3 seconds and a platelet count of less than $50,000 / \mathrm{mm} 3$ are considered contraindications to elective surgery (13). In addition to increased bleeding risk is reported in patients with INR greater than 1.5 compared with patients with INR of 1.3 to 1.5 and 1.5to 2.0-fold the mean reference range value of aPTT has been recommended as a trigger for coagulation factor replacement $(4,13)$.

Major intra-operative blood loss is correlated significantly with postoperative coagulopathy. Coagulation disorder observed immediately after RLLR may be related mostly to blood loss and to the diluting effect of the intraoperative infused fluids, although the extent of the resection appears to be the most important factor in the extension of the PT, aPTT, INR observed from POD 1 (Figure 1). Thorough intra-operative hemostasis is very important and must be understand before the surgery is concluded. Expansion of the circulating blood volume and transfusion of fresh blood should be carried routinely once a coagulation disorder is confirmed, and prompt administration of fibrinogen, prothrombin complex, fresh platelets, and plasma cold precipitates also is important (8). Careful and minimize liver manipulation during RLLR, and thorough hemostasis and drainage are crucial for success in achieving hemostasis.

Current pharmacological, surgical, and radiological methods are available for controlling hemorrhage and achieving effective hemostasis during RLLR. Advanced surgical techniques and growing experience could decrease blood loss, diminishing the effects on coagulation disorders. Since, for many years, the surgical technique for RLLR has been standardized in our center, the effects of surgical trauma may therefore, be considered to be similar for all RLLR. Intra-operative blood loss and infused fluids were observed similar to each patients in our study. Hence, MLR volume appears to be the most important factor in the extension of the PT, aPTT, and INR observed from POD 0 to POD 2. Therefore, patients who have the risk of post-operative thromboembolic complications should be reassessed after the postoperative second day.

Today, due to careful monitoring and correction of coagulopathy, RLLR is a safe and widely practiced procedure without peri-operative anticoagulant prophylaxis in our center. The reliable surgery for RLLR probably can be improved if the liver transplant surgeons involved have comprehensive knowledge of the expected complications of hemostasis disorders. On the other hand, donor death of post-right liver lobe and left lobe liver donation due to pulmonary embolism (PE) and the morbidity of deep venous thrombosis (DVT) are well documented in the literature (21). The incidence of PE in general surgery patients is estimated from $0.3-1.5 \%$ (22). However, the largest and best study of living liver donation in adult to adult transplant provides the rationale for mandating DVT anticoagulation prophylaxis in living liver donors and this study demonstrated a $0.95 \%$ incidence of PE in healthy donors (21). Also, according to this retrospective uncontrolled study without specific follow up monitoring for DVT and/or silent PE does not allow anticoagulation prophylaxis should not be used. We did not experience any clinical thrombosis or embolism in the 100 donors. The patients after major hepatectomy sometimes have thrombosis without any symptoms and asymptomatic PE are known to occur in peri-operative patients. We could not check deep vein thrombosis at least with Doppler echography routinely. 
Post-operative anticoagulation management following a large abdominal surgery, and especially after major liver resection, is a very crucial topic that absolutely needs to be clarified. This is an important topic, namely the risk of hemorrhage in elective liver resection vs the risk of a hypercoaguable state and a thrombotic complication. Postoperative thrombotic complications are responsible for very significant mortality and morbidity. In the literature, some institutions are started antithrombotic prophylaxis therapy intra-operatively and continued postoperatively for both live liver donors and big liver resection related to other causes. To our knowledge, they have not seen an increase in the rate of postoperative bleeding (23). A recently published survey demonstrated that over 50\% of surgeons disagree with holding antithrombotic prophylaxis until laboratory normalization of coagulation (24). Another publication evaluates efficacy of different techniques of antithrombotic prophylaxis. In the publication, the ratio of post-operative thrombotic complications were evaluated. The authors found that even modern aggressive prophylaxis cannot completely prevent thromboembolism. In their study, prophylaxis was started preoperatively in $68 \%$ of patients. There was a relatively high incidence of thrombotic events in patients who received thrombotic prophylaxis. The authors stated that current strategy might be not aggressive enough (25). According to current information and based on the results, it is not possible to say if prophylaxis is indicated or not, and if it is associated with bleeding complications or not. In our experiences for healty live donors with any risks for hipercoagulation, anticoagulant prophylaxis is not needed in right-lobe donor hepatectomy for LDLT, since there was no thromboembolic event in our series.

Our study had several limitations. Firstly, we did not measure remnant hepatic blood flow and remmnant hepatic volume. Expected remnant hepatic volume is measured by the data of CT volumetry. The change of platelet count and/or PT-INR would depend on the volume of the remnant liver. Secondly, we did not measure more specific hepatic and coagulation function tests. Thirdly, the postoperative follow-up periods should be extended. Fourthly, this retrospective examined only postoperative changes in biochemical and hepatological laboratory values in RLLR. For the high scientific quality, the values should be examined according to the graft type ( ie, right lobe vs left lobe ) or GRWR ( less than $1.0 \%$ vs more than $1.0 \%$ ) and so on. Fifthly, we excluded donors who received any anticoagulant prophylaxis from this research. Sixthly, the correlation between INR and hypercoagulable states in liver surgery and cirrhosis is poor (26). There was no other measurement of the tendency to clot in our patients (such as intra-operative thromboelastography).

In conclusion, liver resection operations, particularly MLR, are different from other operations of general surgery with their effect on coagulation cascade. Patients with MLR run an increased risk of bleeding complications because of underlying coagulopathy. Postoperative coagulation profile associated with amount of hepatic resection and identify risk factors that may predict the development of an abnormal post-operative coagulation profile. Coagulation abnormalities that may be seen in proportion with the liver resection must be taken into consideration in this patient group and routine anticoagulant prophylaxis protocols should be reviewed. Anticoagulant prophylaxis after RLDH should be a routine procedure during the postoperative first and second days when there is not a risk of the postoperative bleeding. On the other hand, anticoagulant prophylaxis after RLDH may not be a routine procedure during the postoperative first and second days when the postoperative bleeding is most hazardous. According to our present work, the anticoagulation prophylaxis would seem to be safer on day 2 postoperative.

Declaration of Interest:-

The authors report no conflict of interest and no funding from any company.

\section{Acknowledgements:-}

The authors want to thank Sezai Yilmaz M.D., Prof. for the materials of study and interpretation of data. 


\section{References:-}

1. Yuan FS, Ng SY, Ho KY et al. Abnormal coagulation profile after hepatic resection: the effect of chronic hepatic disease and implications for epidural analgesia. J Clin Anesth 2012; 24: 398-403.

2. Nguyen KT, Gamblin TC, Geller DA. World review of laparoscopic liver resection-2,804 patients. Ann Surg 2009; 250: 831-41.

3. Endo T, Amemiya N, Kume S. Disorders of coagulation/fibrinolysis and bleeding tendency. Nihon Rinsho 1994; 52: 119-23.

4. Kim YK, Shin WJ, Song JG et al. Factors associated with changes in coagulation profiles after living donor hepatectomy. Transplant Proc 2010; 42: 2430-5.

5. Hatipoglu S, Bulbuloglu B, Piskin T, Kayaalp C, Yilmaz S. Living donor liver transplantation for alveolar echinococcus is a difficult procedure. Transplant Proc 2013; 45: 1028-30.

6. Hatipoglu S, Olmez A, Ozgor D, Kayaalp C, Yilmaz S. Living donor liver transplantation in the absence of inferior vena cava: a case report. Transplant Proc 2012; 44: 1761-3.

7. Hatipoglu S, Bulbuloglu E, Ates M, Kayaalp C, Yilmaz S. Liver transplantation following blunt liver trauma. Transplant Proc 2012; 44: 1720-1.

8. Jin S, Fu Q, Wuyun G, Wuyun T. Management of post-hepatectomy complications. World J Gastroenterol 2013; 19: 7983-91.

9. Silva MA, Muralidharan V, Mirza DF. The management of coagulopathy and blood loss in liver surgery. Semin Hematol 2004; $41: 132-9$.

10. Lau AW, Chen CC, Wu RS, Poon KS. Hypothermia as a cause of coagulopathy during hepatectomy. Acta Anaesthesiol Taiwan 2010; 48:103-6.

11. Sabate A, Dalmau A, Koo M, Aparicio I, Costa M, Contreras L. Coagulopathy management in liver transplantation. Transplant Proc 2012; 44: 1523-5.

12. Weinberg L, Scurrah N, Gunning K, McNicol L. Postoperative changes in prothrombin time following hepatic resection: implications for perioperative analgesia. Anaesth Intensive Care 2006; 34: 438-43.

13. Amitrano L, Guardascione MA, Brancaccio V, Balzano A. Coagulation disorders in liver disease. Semin Liver Dis 2002; 22: 83-96.

14. Ko JS, Gwak MS, Choi SJ et al. The effects of desflurane and propofol-remifentanil on postoperative hepatic and renal functions after right hepatectomy in liver donors. Liver Transpl 2008; 14: 1150.

15. Ko JS, Gwak MS, Choi SJ et al. The effects of desflurane and sevoflurane on hepatic and renal functions after right hepatectomy in living donors. Transpl Int 2010; 23: 736.

16. Siniscalchi A, Begliomini B, De Pietri L et al. Increased prothrombin time and platelet counts in living donor right hepatectomy: implications for epidural anesthesia. Liver Transpl 2004; 10: 1144-9.

17. Borromeo CJ, Stix MS, Lally A, Pomfret EA. Epidural catheter and increased prothrombin time after right lobe hepatectomy for living donor liver transplantation. Anesth Analg 2000; 91: 1139-41.

18. Tsui SL, Yong BH, Ng KF et al. Delayed epidural catheter removal: the impact of postoperative coagulopathy. Anaesth Intensive Care 2004; 32: 630-6.

19. Schumann R, Zabala L, Angelis M, et al. Altered hematologic profiles following donor right hepatectomy and implications for perioperative analgesic management. Liver Transpl 2004;10: 363-8.

20. Choi SJ, Gwak MS, Ko JS et al. The changes in coagulation profile and epidural catheter safety for living liver donors: a report on 6 years of our experience. Liver Transpl 2007;13: 62-70.

21. Abecassis MM, Fisher RA, Olthoff KM, et al; A2ALL Study Group. Complications of living donor hepatic lobectomy-a comprehensive report. Am J Transplant 2012; 12:1208-17.

22. Desciak MC, Martin DE. Perioperative pulmonary embolism: diagnosis and anesthetic management. J Clin Anesth 2011; 23: 153-65.

23. Yoo T, Kim SH, Kim YK, Cho SY, Park SJ. Low-dose heparin therapy during living donor right hepatectomy is associated with few side effects and does not increase vascular thrombosis in liver transplantation. Transplant Proc 2013; 45: 222-4.

24. Weiss MJ, Kim Y, Ejaz A, et al. Venous thromboembolic prophylaxis after a hepatic resection: patterns of care among liver surgeons. HPB 2014; 16: 892-8.

25. Ejaz A, Spolverato G, Kim Y, et al. Defining incidence and risk factors of venous thromboemolism after hepatectomy. J Gastrointest Surg 2014;18: 1116-24.

26. Bezeaud A, Denninger MH, Dondero F, et al. Hypercoagulability after partial liver resection. Thromb Haemost 2007; 98: 1252-6. 